\title{
Note on a $q$-Contour Integral Formula of Gasper-Rahman
}

\author{
Jian-Ping Fang \\ School of Mathematical Science, Huaiyin Normal University, Huaian, Jiangsu 223300, China \\ Correspondence should be addressed to Jian-Ping Fang; fjp7402@163.com
}

Received 23 May 2013; Accepted 6 August 2013

Academic Editor: Shanhe Wu

Copyright (C) 2013 Jian-Ping Fang. This is an open access article distributed under the Creative Commons Attribution License, which permits unrestricted use, distribution, and reproduction in any medium, provided the original work is properly cited.

We use the $q$-Chu-Vandermonde formula and transformation technique to derive a more general $q$-integral equation given by Gasper and Rahman, which involves the Cauchy polynomial. In addition, some applications of the general formula are presented in this paper.

\section{Introduction and Main Result}

It is well known that the $q$-integral is an important branch of $q$-series theory. There are many techniques to achieve the ends; for instance, combinatorics method (cf. [1]), analysis methods (cf. [2-4]), and method of transformation (cf. [5-7]) are usually used. In 1989, Gasper and Rahman applied some analysis techniques to derive the following $q$-contour integral formula (cf. [8, Equation (3.17)]):

$$
\begin{aligned}
& \frac{1}{2 \pi i} \int_{C} \frac{(\gamma / z, b q z, q z / \gamma ; q)_{\infty}}{(\alpha / z, a z, b z ; q)_{\infty}}\left(q z / \gamma q^{m_{0}} ; q\right)_{m_{0}} \\
& \quad \times \prod_{i=1}^{r}\left(a_{i} z ; q\right)_{m_{i}} \frac{d z}{z} \\
& =\frac{(\gamma / \alpha, \alpha q / \gamma, q b / a ; q)_{\infty}}{(a \alpha, q / a \alpha, b \alpha ; q)_{\infty}}\left(q / b \gamma q^{m_{0}} ; q\right)_{m_{0}}(b \alpha)^{\sum_{i=0}^{r} m_{i}} \\
& \quad \times \prod_{i=1}^{r}\left(a_{i} / b ; q\right)_{m_{i}} .
\end{aligned}
$$

Inspired by $[7,8]$, we employ the above equation and transformation technique to derive a more general $q$-contour integral equation. The main result of this paper is stated as follows.
Theorem 1. If $m_{0}, m_{1}, \ldots, m_{r}$, and $h$ are nonnegative integers and $q=a \gamma q^{\sum_{i=0}^{r} m_{i}}$, then

$$
\begin{aligned}
\frac{1}{2 \pi i} \int_{C} \frac{(\gamma / z, b q z, q z / \gamma ; q)_{\infty}}{(\alpha / z, a z, b z ; q)_{\infty}}\left(q z / \gamma q^{m_{0}} ; q\right)_{m_{0}} \\
\quad \times \prod_{i=1}^{r}\left(a_{i} z ; q\right)_{m_{i}} \prod_{j=1}^{h+1} P_{n_{j}}\left(1 / z ; d_{j}\right) \frac{d z}{z} \\
=\frac{\prod_{l=0}^{h}\left(\alpha d_{l} ; q\right)_{n_{l}}}{\alpha \sum_{i=0}^{h} n_{i}} \frac{(\gamma / \alpha, q \alpha / \gamma, q b / a ; q)_{\infty}}{(a \alpha, q / a \alpha, b \alpha ; q)_{\infty}} \\
\quad \times\left(q / b \gamma q^{m_{0}} ; q\right)_{m_{0}}(b \alpha)^{\sum_{i=0}^{r} m_{i}} \\
\quad \times \prod_{j=1}^{r}\left(a_{j} / b ; q\right)_{m_{j}} \\
\quad \times \prod_{i=0}^{h} \sum_{k_{i}=0}^{n_{i}} \frac{\left(q^{-n_{i}}, q^{A_{i}} b \alpha, q^{A_{i}+n_{i+1}} d_{i+1} \alpha, \ldots, q^{A_{i}+n_{h}} d_{h} \alpha ; q\right)_{k_{i}}}{\left(q, q^{A_{i}} d_{i} \alpha, q^{A_{i}} d_{i+1} \alpha, \ldots, q^{A_{i}} d_{h} \alpha ; q\right)_{k_{i}}} \\
\quad \times q^{k_{i}\left(1-\sum_{j=i+1}^{h} n_{j}\right)},
\end{aligned}
$$

provided that $|\gamma / \alpha|<1$ and $C$ is a deformation of the unit circle so that the poles of $1 /(a z, b z ; q)_{\infty}$ lie outside the contour and the origin and the poles of $1 /(\alpha / z ; q)_{\infty}$ lie inside the contour. 
Where $P_{n}(a ; b)$ denotes the Cauchy polynomial defined as (7), one denotes that $A_{i}=\sum_{j=0}^{i-1} k_{j}$, and when $i=0$, one sets one $A_{0}=\sum_{j=0}^{i-1} k_{j}=0$.

\section{Notations and Lemmas}

We adopt the custom notations given in [9]. It is supposed that $0<|q|<1$ in this paper. We use $N$ to denote the set of all nonnegative integers.

For any complex parameter $a$, the $q$-shifted factorials are defined as

$$
\begin{gathered}
(a ; q)_{0}=1,(a ; q)_{n}=\prod_{k=0}^{n-1}\left(1-a q^{k}\right), \quad n=1,2, \ldots \\
(a ; q)_{\infty}=\prod_{k=0}^{\infty}\left(1-a q^{k}\right) .
\end{gathered}
$$

For brevity, we also use the following notation:

$$
\left(a_{1}, a_{2}, \ldots, a_{m} ; q\right)_{n}=\left(a_{1} ; q\right)_{n}\left(a_{2} ; q\right)_{n} \cdots\left(a_{m} ; q\right)_{n} .
$$

The $q$-binomial coefficient and the $q$-binomial theorem are given by

$$
\begin{gathered}
{\left[\begin{array}{l}
n \\
k
\end{array}\right]=\frac{(q ; q)_{n}}{(q ; q)_{k}(q ; q)_{n-k}},} \\
\sum_{n=0}^{\infty} \frac{(a ; q)_{n} x^{n}}{(q ; q)_{n}}=\frac{(a x ; q)_{\infty}}{(x ; q)_{\infty}}, \quad|x|<1 .
\end{gathered}
$$

The basic hypergeometric series ${ }_{s} \Phi_{t}$ is given by

$$
\begin{aligned}
{ }_{s} \Phi_{t}\left(\begin{array}{c}
a_{1}, a_{2}, \ldots, a_{s} \\
\left.b_{1}, b_{2}, \ldots, b_{t} ; q, x\right)
\end{array}\right. \\
\quad=\sum_{k=0}^{\infty} \frac{\left(a_{1}, a_{2} \ldots, a_{s} ; q\right)_{k}}{\left(q, b_{1}, \ldots, b_{t} ; q\right)_{k}}\left[(-1)^{k} q^{\left.\left(\begin{array}{l}
k \\
2
\end{array}\right)\right]^{1+t-s} x^{k} .}\right.
\end{aligned}
$$

In this paper, we denote that $\left(\begin{array}{l}n \\ 2\end{array}\right)=n(n-1) / 2$ and $k, m, n, s$, $t \in N$.

Let $a, b$ be any complex variables; then, the Cauchy polynomial $P_{n}(a ; b)$ is defined as

$$
\begin{array}{r}
P_{0}(a ; b)=1, \quad P_{n}(a ; b)=(a-b)(a-b q) \cdots\left(a-b q^{n-1}\right) \\
n \geq 1 .
\end{array}
$$

Recall that $q$-Chu-Vandermonde's identity (cf. [9, page 14, Equation (1.5.3)] ) is given as follows:

$$
{ }_{2} \Phi_{1}\left(\begin{array}{cc}
q^{-n}, & a \\
f
\end{array} ; q, q\right)=\frac{a^{n}(f / a ; q)_{n}}{(f ; q)_{n}} .
$$

As we know, it is one of the fundamental formulas in the basic hypergeometric series. Some applications of it were introduced in $[5,10,11]$. We will apply this identity to start our proof in the following. Since we assume that the integrals are the same established condition as the theorem, we omit the condition in the following.
Lemma 2. One has

$$
\begin{aligned}
& \frac{1}{2 \pi i} \int_{C} \frac{(\gamma / z, b q z, q z / \gamma ; q)_{\infty}}{(\alpha / z, a z, b z ; q)_{\infty}}\left(q z / \gamma q^{m_{0}} ; q\right)_{m_{0}} \\
& \times \prod_{i=1}^{r}\left(a_{i} z ; q\right)_{m_{i}} \frac{P_{n}(1 / z ; f)}{z} d z \\
&= \frac{(f \alpha ; q)_{n}}{\alpha^{n}} \frac{(\gamma / \alpha, \alpha q / \gamma, q b / a ; q)_{\infty}}{(a \alpha, q / a \alpha, b \alpha ; q)_{\infty}}\left(q / b \gamma q^{m_{0}} ; q\right)_{m_{0}} \\
& \quad \times(b \alpha)^{\sum_{i=0}^{r} m_{i}} \prod_{i=1}^{r}\left(a_{i} / b ; q\right)_{m_{i}} \\
& \quad \times \sum_{k=0}^{n} \frac{\left(q^{-n}, b \alpha ; q\right)_{k}\left(a \gamma q^{\sum_{i=0}^{r} m_{i}}\right)^{k}}{(q, f \alpha ; q)_{k}} .
\end{aligned}
$$

Proof. We rewrite (8) as follows:

$$
\sum_{k=0}^{n} \frac{\left(q^{-n} ; q\right)_{k}}{(q, f ; q)_{k}} q^{k} \frac{1}{\left(a q^{k} ; q\right)_{\infty}}=\frac{a^{n}(f / a ; q)_{n}}{(f ; q)_{n}(a ; q)_{\infty}} .
$$

Replacing $(a, c)$ by $(\alpha / z, f \alpha)$, respectively, we have

$$
\sum_{k=0}^{n} \frac{\left(q^{-n} ; q\right)_{k}}{(q, f \alpha ; q)_{k}} q^{k} \frac{1}{\left(q^{k} \alpha / z ; q\right)_{\infty}}=\frac{\alpha^{n}}{(f \alpha ; q)_{n}} \frac{P_{n}(1 / z ; f)}{(\alpha / z ; q)_{\infty}} .
$$

Both sides of (11) multiply by

$$
\frac{(\gamma / z, b q z, q z / \gamma ; q)_{\infty}}{(a z, b z ; q)_{\infty}}\left(q z / \gamma q^{m_{0}} ; q\right)_{m_{0}} \prod_{i=1}^{r}\left(a_{i} z ; q\right)_{m_{i}} \frac{1}{z} .
$$

Then, we have

$$
\begin{aligned}
\sum_{k=0}^{n} \frac{\left(q^{-n} ; q\right)_{k} q^{k}}{(q, f \alpha ; q)_{k}} \frac{(\gamma / z, b q z, q z / \gamma ; q)_{\infty}}{\left(q^{k} \alpha / z, a z, b z ; q\right)_{\infty}}\left(q z / \gamma q^{m_{0}} ; q\right)_{m_{0}} \\
\quad \times \prod_{i=1}^{r}\left(a_{i} z ; q\right)_{m_{i}} \frac{1}{z} \\
=\frac{\alpha^{n}}{(f \alpha ; q)_{n}} \frac{(\gamma / z, b q z, q z / \gamma ; q)_{\infty}}{(\alpha / z, a z, b z ; q)_{\infty}}\left(q z / \gamma q^{m_{0}} ; q\right)_{m_{0}} \\
\quad \times \prod_{i=1}^{r}\left(a_{i} z ; q\right)_{m_{i}} \frac{P_{n}(1 / z ; f)}{z} .
\end{aligned}
$$

Taking the $q$-integral on both sides of (13) with respect to variable $z$, we get

$$
\begin{aligned}
\sum_{k=0}^{n} \frac{\left(q^{-n} ; q\right)_{k} q^{k}}{(q, f \alpha ; q) k} \int_{C} & \frac{(\gamma / z, b q z, q z / \gamma ; q)_{\infty}}{\left(q^{k} \alpha / z, a z, b z ; q\right)_{\infty}} \\
& \times\left(q z / \gamma q^{m_{0}} ; q\right)_{m_{0}} \prod_{i=1}^{r}\left(a_{i} z ; q\right)_{m_{i}} \frac{1}{z} d z \\
=\frac{\alpha^{n}}{(f \alpha ; q)_{n}} \int_{C} \frac{(\gamma / z, b q z, q z / \gamma ; q)_{\infty}}{(\alpha / z, a z, b z ; q)_{\infty}} & \times\left(q z / \gamma q^{m_{0}} ; q\right)_{m_{0}} \prod_{i=1}^{r}\left(a_{i} z ; q\right)_{m_{i}} \frac{P_{n}(1 / z ; f)}{z} d z .
\end{aligned}
$$


Employing (1) to the left side of (14), we have the desired result after some simplification. have

On the other hand, if we multiply $(13)$ by $P_{n_{1}}(1 / z ; g)$, we

$$
\begin{aligned}
& \sum_{k=0}^{n} \frac{\left(q^{-n} ; q\right)_{k} q^{k}}{(q, f \alpha ; q)_{k}} \frac{(\gamma / z, b q z, q z / \gamma ; q)_{\infty}}{\left(q^{k} \alpha / z, a z, b z ; q\right)_{\infty}}\left(q z / \gamma q^{m_{0}} ; q\right)_{m_{0}} \\
& \quad \times \prod_{i=1}^{r}\left(a_{i} z ; q\right)_{m_{i}} \frac{P_{n_{1}}(1 / z ; g)}{z} \\
& =\frac{\alpha^{n}}{(f \alpha ; q)_{n}} \frac{(\gamma / z, b q z, q z / \gamma ; q)_{\infty}}{(\alpha / z, a z, b z ; q)_{\infty}}\left(q z / \gamma q^{m_{0}} ; q\right)_{m_{0}} \\
& \quad \times \prod_{i=1}^{r}\left(a_{i} z ; q\right)_{m_{i}} \frac{P_{n_{1}}(1 / z ; g) P_{n}(1 / z ; f)}{z} .
\end{aligned}
$$

Taking the $q$-integral on both sides of (15) with respect to variable $z$, we use (9) in the resulting equation. After simple rearrangements, noting that $q=a \gamma q^{\sum_{i=0}^{r} m_{i}}$, we get the following.

\section{Lemma 3. One has}

$$
\begin{aligned}
& \frac{1}{2 \pi i} \int_{C} \frac{(\gamma / z, b q z, q z / \gamma ; q)_{\infty}}{(\alpha / z, a z, b z ; q)_{\infty}}\left(q z / \gamma q^{m_{0}} ; q\right)_{m_{0}} \\
& \times \prod_{i=1}^{r}\left(a_{i} z ; q\right)_{m_{i}} \frac{P_{n_{1}}(1 / z ; g) P_{n}(1 / z ; f)}{z} d z \\
&= \frac{(g \alpha ; q)_{n_{1}}(f \alpha ; q)_{n}}{\alpha^{n_{1}} \alpha^{n}} \frac{(\gamma / \alpha, q \alpha / \gamma, q b / a ; q)_{\infty}}{(a \alpha, q / a \alpha, b \alpha ; q)_{\infty}} \\
& \quad \times\left(q / b \gamma q^{m_{0}} ; q\right)_{m_{0}}(b \alpha)^{\sum_{i=0}^{r} m_{i}} \prod_{i=1}^{r}\left(a_{i} / b ; q\right)_{m_{i}} \\
& \quad \times \sum_{k=0}^{n} \frac{\left(q^{-n}, b \alpha, q^{n_{1}} g \alpha ; q\right)_{k} q^{k\left(1-n_{1}\right)}}{(q, f \alpha, g \alpha ; q)_{k}} \\
& \quad \times \sum_{k_{1}=0}^{n_{1}} \frac{\left(q^{-n_{1}}, b \alpha q^{k} ; q\right)_{k_{1}} q^{k_{1}}}{\left(q, g \alpha q^{k} ; q\right)_{k_{1}}} .
\end{aligned}
$$

Both sides of (11) multiply by

$$
\begin{aligned}
& \frac{(\gamma / z, b q z, q z / \gamma ; q)_{\infty}}{(a z, b z ; q)_{\infty}}\left(q z / \gamma q^{m_{0}} ; q\right)_{m_{0}} \prod_{i=1}^{r}\left(a_{i} z ; q\right)_{m_{i}} \\
& \quad \times \prod_{j=1}^{h} P_{n_{j}}\left(1 / z ; d_{j}\right) \frac{1}{z} .
\end{aligned}
$$

Then, taking the $q$-integral on both sides of the result equation with respect to variable $z$, we find the following.
Lemma 4. On has

$$
\begin{gathered}
\int_{C} \frac{(\gamma / z, b q z, q z / \gamma ; q)_{\infty}}{(\alpha / z, a z, b z ; q)_{\infty}}\left(q z / \gamma q^{m_{0}} ; q\right)_{m_{0}} \prod_{i=1}^{r}\left(a_{i} z ; q\right)_{m_{i}} \\
\times \prod_{j=1}^{h+1} P_{n_{j}}\left(1 / z ; d_{j}\right) \frac{d z}{z} \\
=\frac{\left(\alpha d_{h+1} ; q\right)_{n_{h+1}}}{\alpha^{n_{h+1}}} \\
\quad \times \sum_{k=0}^{n_{h+1}} \frac{\left(q^{-n_{h+1}} ; q\right)_{k} q^{k}}{\left(q, \alpha d_{h+1} ; q\right)_{k}} \\
\times \int_{C} \frac{(\gamma / z, b q z, q z / \gamma ; q)_{\infty}}{\left(q^{k} \alpha / z, a z, b z ; q\right)_{\infty}}\left(q z / \gamma q^{m_{0}} ; q\right)_{m_{0}} \\
\quad \times \prod_{i=1}^{r}\left(a_{i} z ; q\right)_{m_{i}} \prod_{j=1}^{h} P_{n_{j}}\left(1 / z ; d_{j}\right) \frac{d z}{z}
\end{gathered}
$$

where $\left(n_{h+1}, d_{h+1}\right)$ denote $(n, f)$, respectively.

\section{Proof and Some Applications}

Now, we return to the proof of Theorem 1.

The following result can be easily derived from (16) and (18):

$$
\begin{aligned}
\int_{C} \frac{(\gamma / z, b q z, q z / \gamma ; q)_{\infty}}{(\alpha / z, a z, b z ; q)_{\infty}}\left(q z / \gamma q^{m_{0}} ; q\right)_{m_{0}} \\
\times \prod_{i=1}^{r}\left(a_{i} z ; q\right)_{m_{i}} \prod_{j=1}^{3} P_{n_{j}}\left(1 / z ; d_{j}\right) \frac{d z}{z} \\
=\frac{\prod_{i=1}^{3}\left(\alpha d_{i} ; q\right)_{n_{i}} \frac{(\gamma / \alpha, q \alpha / \gamma, q b / a ; q)_{\infty}}{(a \alpha, q / a \alpha, b \alpha ; q)_{\infty}}}{\alpha \sum_{i=1}^{3} n_{i}} \\
\quad \times\left(q / b \gamma q^{m_{0}} ; q\right)_{m_{0}}(b \alpha)^{\sum_{i=0}^{r} m_{i}} \prod_{j=1}^{r}\left(a_{j} / b ; q\right)_{m_{j}} \\
\quad \times \sum_{k=0}^{n} \frac{\left(q^{-n}, b \alpha, q^{n_{1}} d_{1} \alpha, q^{n_{2}} d_{2} \alpha ; q\right)_{k}}{\left(q, f \alpha, d_{1} \alpha, d_{2} \alpha ; q\right)_{k}} q^{k\left(1-n_{1}-n_{2}\right)} \\
\quad \times \sum_{k_{1}=0}^{n_{1}} \frac{\left(q^{-n_{1}}, q^{k} b \alpha, q^{n_{2}+k} d_{2} \alpha ; q\right)_{k_{1}}}{\left(q, q^{k} d_{1} \alpha, q^{k} d_{2} \alpha ; q\right)_{k_{1}}} q^{k_{1}\left(1-n_{2}\right)} \\
\quad \times \sum_{k_{2}=0}^{n_{2}} \frac{\left(q^{-n_{2}}, q^{k+k_{1}} b \alpha ; q\right)_{k_{2}}}{\left(q, q^{k+k_{1}} d_{2} \alpha ; q\right)_{k_{2}}} q^{k_{2}} .
\end{aligned}
$$

Letting $n=n_{0}, k=k_{0}$, and $f=d_{0}$ and combining (19) with (18), by induction, similar proof can be performed to get the desired result.

Taking $n_{1}=n_{2}=\cdots=n_{h+1}=0$ in (2), the theorem goes back to formula (1). Putting $n_{1}=\cdots=n_{h}=0$ in (2), we have the following. 
Corollary 5. One has

$$
\begin{aligned}
\frac{1}{2 \pi i} \int_{C} \frac{(\gamma / z, b q z, q z / \gamma ; q)_{\infty}}{(\alpha / z, a z, b z ; q)_{\infty}}\left(q z / \gamma q^{m_{0}} ; q\right)_{m_{0}} \\
\quad \times \prod_{i=1}^{r}\left(a_{i} z ; q\right)_{m_{i}} \frac{P_{n}\left(1 / z ; d_{0}\right)}{z} d z \\
=\frac{(\gamma / \alpha, \alpha q / \gamma, q b / a ; q)_{\infty}}{(a \alpha, q / a \alpha, b \alpha ; q)_{\infty}}\left(q / b \gamma q^{m_{0}} ; q\right)_{m_{0}}(b \alpha)^{\sum_{i=0}^{r} m_{i}} \\
\quad \times \prod_{i=1}^{r}\left(a_{i} / b ; q\right)_{m_{i}}\left(d_{0} / b ; q\right)_{n} b^{n} .
\end{aligned}
$$

Letting $n_{2}=\cdots=n_{h}=0$ in (2), we get the following.

Corollary 6. One has

$$
\begin{aligned}
& \frac{1}{2 \pi i} \int_{C} \frac{(\gamma / z, b q z, q z / \gamma ; q)_{\infty}}{(\alpha / z, a z, b z ; q)_{\infty}}\left(q z / \gamma q^{m_{0}} ; q\right)_{m_{0}} \\
& \times \prod_{i=1}^{r}\left(a_{i} z ; q\right)_{m_{i}} \frac{P_{n_{1}}\left(1 / z ; d_{1}\right) P_{n}\left(1 / z ; d_{0}\right)}{z} d z \\
&= \frac{(\gamma / \alpha, \alpha q / \gamma, q b / a ; q)_{\infty}}{(a \alpha, q / a \alpha, b \alpha ; q)_{\infty}}\left(q / b \gamma q^{m_{0}} ; q\right)_{m_{0}}(b \alpha)^{\sum_{i=0}^{r} m_{i}} \\
& \quad \times \prod_{i=1}^{r}\left(a_{i} / b ; q\right)_{m_{i}}\left(d_{0} / b ; q\right)_{n}\left(d_{1} / b ; q\right)_{n_{1}} b^{n+n_{1}}
\end{aligned}
$$

Combining (21) with (18), by induction and applying (2), we can conclude the following.

Theorem 7. One has

$$
\begin{aligned}
& \frac{1}{2 \pi i} \int_{C} \frac{(\gamma / z, b q z, q z / \gamma ; q)_{\infty}}{(\alpha / z, a z, b z ; q)_{\infty}}\left(q z / \gamma q^{m_{0}} ; q\right)_{m_{0}} \\
& \times \prod_{i=1}^{r}\left(a_{i} z ; q\right)_{m_{i}} \prod_{j=0}^{h} P_{n_{j}}\left(1 / z ; d_{j}\right) \frac{d z}{z} \\
&= \frac{(\gamma / \alpha, q \alpha / \gamma, q b / a ; q)_{\infty}}{(a \alpha, q / a \alpha, b \alpha ; q)_{\infty}}\left(q / b \gamma q^{m_{0}} ; q\right)_{m_{0}}(b \alpha)^{\sum_{i=0}^{r} m_{i}} b^{\sum_{i=0}^{h} n_{i}} \\
& \quad \times \prod_{i=1}^{r}\left(a_{i} / b ; q\right)_{m_{i}} \prod_{j=0}^{h}\left(d_{j} / b ; q\right)_{n_{j}} .
\end{aligned}
$$

Comparing (2) and (22), we have the following interesting identity.
Corollary 8. If $m_{0}, m_{1}, \ldots, m_{r}$, and $h$ are nonnegative integers, then

$$
\begin{aligned}
& \sum_{k_{0}=0}^{n_{0}} \frac{\left(q^{-n_{0}}, b \alpha, q^{n_{1}} d_{1} \alpha, \ldots, q^{n_{h}} d_{h} \alpha ; q\right)_{k_{0}}}{\left(q, d_{0} \alpha, d_{1} \alpha, \ldots, d_{h} \alpha ; q\right)_{k_{0}}} q^{k_{0}\left(1-\sum_{j=1}^{h} n_{j}\right)} \\
& \quad \times \prod_{i=1}^{h} \sum_{k_{i}=0}^{n_{i}} \frac{\left(q^{-n_{i}}, q^{A_{i}} b \alpha, q^{A_{i}+n_{i+1}} d_{i+1} \alpha, \ldots, q^{A_{i}+n_{h}} d_{h} \alpha ; q\right)_{k_{i}}}{\left(q, q^{A_{i}} d_{i} \alpha, q^{A_{i}} d_{i+1} \alpha, \ldots, q^{A_{i}} d_{h} \alpha ; q\right)_{k_{i}}} \\
& \quad \times q^{k_{i}\left(1-\sum_{j=i+1}^{h} n_{j}\right)} \\
& =\prod_{i=0}^{h} \frac{\left(d_{i} / b ; q\right)_{n_{i}}}{\left(d_{i} \alpha ; q\right)_{n_{i}}}(b \alpha)^{n_{0}+n_{1}+\cdots+n_{h}} .
\end{aligned}
$$

Taking $h=1$ and $d_{0}=d_{1}=q b$ in (23), we have

$$
\begin{gathered}
\sum_{k_{0}=0}^{n_{0}}\left[\begin{array}{l}
n_{0} \\
k_{0}
\end{array}\right] \frac{\left(b \alpha, q^{n_{1}+1} b \alpha ; q\right)_{k_{0}}}{(q b \alpha, q b \alpha ; q)_{k_{0}}}(-1)^{k_{0}} q^{\left(\begin{array}{c}
k_{0}+1 \\
2
\end{array}\right)-k_{0}\left(n_{0}+n_{1}\right)} \\
\times \sum_{k_{1}=0}^{n_{1}}\left[\begin{array}{l}
n_{1} \\
k_{1}
\end{array}\right] \frac{\left(q^{k_{0}} b \alpha ; q\right)_{k_{1}}}{\left(q^{k_{0}+1} b \alpha ; q\right)_{k_{1}}}(-1)^{k_{1}} q^{\left(\begin{array}{c}
k_{1}+1 \\
2
\end{array}\right)-k_{1} n_{1}} \\
=\frac{(q ; q)_{n_{0}}(q ; q)_{n_{1}}}{(q b \alpha ; q)_{n_{0}}(q b \alpha ; q)_{n_{1}}}(b \alpha)^{n_{0}+n_{1}} .
\end{gathered}
$$

Setting $b \alpha=q$, then letting $q \rightarrow 1$ in the above identity, we have the following.

Corollary 9. If $n_{0}, n_{1} \in N$, then

$$
\begin{gathered}
\sum_{k_{0}=0}^{n_{0}}\left(\begin{array}{l}
n_{0} \\
k_{0}
\end{array}\right) \frac{\left(n_{1}+2\right)_{k_{0}}}{(2)_{k_{0}}}(-1)^{k_{0}} \sum_{k_{1}=0}^{n_{1}}\left(\begin{array}{l}
n_{1} \\
k_{1}
\end{array}\right) \frac{1}{k_{0}+k_{1}+1}(-1)^{k_{1}} \\
=\frac{1}{\left(n_{0}+1\right)\left(n_{1}+1\right)},
\end{gathered}
$$

where $(a)_{0}=1$ and $(a)_{n}=a(a+1) \cdots(a+n-1), n \geq 1, n \in N$.

Taking $h=2$ and $d_{0}=d_{1}=d_{2}=q b$ in (23), we have

$$
\sum_{k=0}^{n_{0}}\left[\begin{array}{l}
n_{0} \\
k_{0}
\end{array}\right] \frac{\left(b \alpha, q^{n_{1}+1} b \alpha, q^{n_{2}+1} b \alpha ; q\right)_{k_{0}}}{(q b \alpha, q b \alpha, q b \alpha ; q)_{k_{0}}}(-1)^{k_{0}}
$$

$$
\times q^{\left(\begin{array}{c}
k_{0}+1 \\
2
\end{array}\right)-k_{0}\left(n_{0}+n_{1}+n_{2}\right)}
$$




$$
\begin{aligned}
\times \sum_{k_{1}=0}^{n_{1}}\left[\begin{array}{l}
n_{1} \\
k_{1}
\end{array}\right] \frac{\left(q^{k_{0}} b \alpha, q^{n_{2}+k_{0}+1} b \alpha ; q\right)_{k_{1}}}{\left(q^{k_{0}+1} b \alpha, q^{k_{0}+1} b \alpha ; q\right)_{k_{1}}}(-1)^{k_{1}} \\
\times q^{\left(\begin{array}{c}
k_{1}+1 \\
2
\end{array}\right)-k_{1}\left(n_{1}+n_{2}\right)} \\
\times \sum_{k_{2}=0}^{n_{2}}\left[\begin{array}{l}
n_{2} \\
k_{2}
\end{array}\right] \frac{\left(q^{k_{0}+k_{1}} b \alpha ; q\right)_{k_{2}}}{\left(q^{k_{0}+k_{1}+1} b \alpha ; q\right)_{k_{2}}}(-1)^{k_{2}} \\
\times q^{\left(\begin{array}{c}
k_{2}+1 \\
2
\end{array}\right)-k_{2} n_{2}} \\
=\frac{(q ; q)_{n_{0}}(q ; q)_{n_{1}}(q ; q)_{n_{2}}}{(q b \alpha ; q)_{n_{0}}(q b \alpha ; q)_{n_{1}}(q b \alpha ; q)_{n_{2}}}(b \alpha)^{n_{0}+n_{1}+n_{2}} .
\end{aligned}
$$

Setting $b \alpha=q$, then letting $q \rightarrow 1$ in the above identity, we have the following.

Corollary 10. If $n_{0}, n_{1}, n_{2} \in N$, then

$$
\begin{gathered}
\sum_{k_{0}=0}^{n_{0}}\left(\begin{array}{l}
n_{0} \\
k_{0}
\end{array}\right) \frac{\left(n_{1}+2\right)_{k_{0}}\left(n_{2}+2\right)_{k_{0}}}{(2)_{k_{0}}(2)_{k_{0}}}(-1)^{k_{0}} \\
\times \sum_{k_{1}=0}^{n_{1}}\left(\begin{array}{l}
n_{1} \\
k_{1}
\end{array}\right) \frac{\left(n_{2}+k_{0}+2\right)_{k_{1}}}{\left(k_{0}+2\right)_{k_{1}}}(-1)^{k_{1}} \\
\times \sum_{k_{2}=0}^{n_{2}}\left(\begin{array}{l}
n_{2} \\
k_{2}
\end{array}\right) \frac{1}{k_{0}+k_{1}+k_{2}+1}(-1)^{k_{2}} \\
=\frac{1}{\left(n_{0}+1\right)\left(n_{1}+1\right)\left(n_{2}+1\right)}
\end{gathered}
$$

where $(a)_{0}=1$ and $(a)_{n}=a(a+1) \cdots(a+n-1), n \geq 1, n \in N$.

More general, we have the following identity.

Corollary 11. If $h, n_{0}, n_{1}, \ldots, n_{h} \in N$, then

$$
\begin{gathered}
\sum_{k_{0}, \ldots, k_{h}} \prod_{i=0}^{h-1}\left(\begin{array}{l}
n_{i} \\
k_{i}
\end{array}\right) \frac{\left(A_{i}+n_{i+1}+2\right)_{k_{i}} \cdots\left(A_{i}+n_{h}+2\right)_{k_{i}}}{\left(A_{i}+2\right)_{k_{i}} \cdots\left(A_{i}+2\right)_{k_{i}}} \\
\times \frac{(-1)^{k_{0}+\cdots+k_{h}}}{A_{h}+k_{h}+2}=\prod_{i=0}^{h} \frac{1}{\left(n_{i}+1\right)}
\end{gathered}
$$

where $0 \leq k_{i} \leq n_{i}, i=0, \ldots, h$.

Both sides of (20) multiply by $1 /(q ; q)_{n}$; then, summing $n$ from 0 to $\infty$ and using the $q$-binomial theorem, we find the following.
Corollary 12. If $\max \{|1 / z|,|b|\}<1$, then

$$
\begin{aligned}
\frac{1}{2 \pi i} \int_{C} \frac{(\gamma / z, b q z, q z / \gamma ; q)_{\infty}}{(\alpha / z, 1 / z, a z, b z ; q)_{\infty}}\left(q z / \gamma q^{m_{0}} ; q\right)_{m_{0}} \\
\quad \times \prod_{i=1}^{r}\left(a_{i} z ; q\right)_{m_{i}} \frac{d z}{z} \\
=\frac{(\gamma / \alpha, \alpha q / \gamma, q b / a ; q)_{\infty}}{(a \alpha, q / a \alpha, b \alpha, b ; q)_{\infty}}\left(q / b \gamma q^{m_{0}} ; q\right)_{m_{0}}(b \alpha)^{\sum_{i=0}^{r} m_{i}} \\
\quad \times \prod_{i=1}^{r}\left(a_{i} / b ; q\right)_{m_{i}} .
\end{aligned}
$$

Remark 13. If $n_{1}=n_{2}=\cdots=n_{h}=0$, identity (23) becomes the $q$-Chu-Vandermonde formula.

\section{Acknowledgments}

The author would like to thank the referees and the editors for their many valuable comments and suggestions. The author would also like to thank Professor Bruce C. Berndt for his help and warm hospitality accorded to him during his visit to UIUC. The author is, moreover, supported by Jiangsu Overseas Research and Training Program for University Prominent Young and Middle-Aged Teachers and Presidents. The author is also supported by the National Natural Science Foundation of China (no. 10971078).

\section{References}

[1] M. E. H. Ismail, D. Stanton, and G. Viennot, "The combinatorics of $q$-Hermite polynomials and the Askey-Wilson integral," European Journal of Combinatorics, vol. 8, no. 4, pp. 379-392, 1987.

[2] G. E. Andrews and R. Askey, "Another q-extension of the beta function," Proceedings of the American Mathematical Society, vol. 81, no. 1, pp. 97-100, 1981.

[3] R. Askey, "The q-gamma and q-beta functions," Applicable Analysis, vol. 8, no. 2, pp. 125-141, 1978.

[4] M. E. H. Ismail and D. R. Masson, " $q$-Hermite polynomials, biorthogonal rational functions, and $q$-beta integrals," Transactions of the American Mathematical Society, vol. 346, no. 1, pp. 63-116, 1994.

[5] Z.-G. Liu, "Some operator identities and $q$-series transformation formulas," Discrete Mathematics, vol. 265, no. 1-3, pp. 119139, 2003.

[6] M.-J. Wang, "A remark on Andrews-Askey integral," Journal of Mathematical Analysis and Applications, vol. 341, no. 2, pp. 14871494, 2008.

[7] M.-J. Wang, "A recurring q-integral formula," Applied Mathematics Letters, vol. 23, no. 3, pp. 256-260, 2010.

[8] G. Gasper and M. Rahman, “ $q$-extensions of Barnes', Cauchy's, and Euler's beta integrals," in Topics in Mathematical Analysis, M. Yh. Rassias, Ed., pp. 294-314, World Scientific, London, UK, 1989.

[9] G. Gasper and M. Rahman, Basic Hypergeometric Series, Cambridge University Press, Cambridge, UK, 2nd edition, 2004. 
[10] J.-P. Fang, " $q$-differential operator identities and applications," Journal of Mathematical Analysis and Applications, vol. 332, no. 2, pp. 1393-1407, 2007.

[11] J.-P. Fang, "Extensions of q-Chu-Vandermonde's identity," Journal of Mathematical Analysis and Applications, vol. 339, no. 2, pp. 845-852, 2008. 


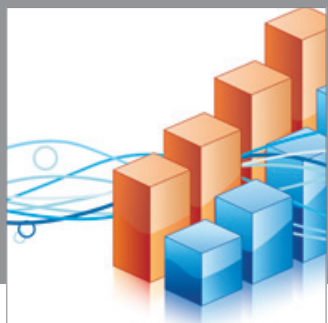

Advances in

Operations Research

mansans

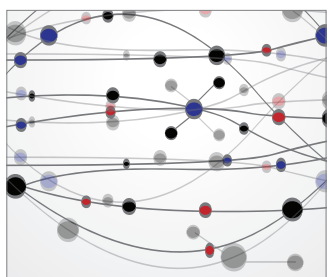

The Scientific World Journal
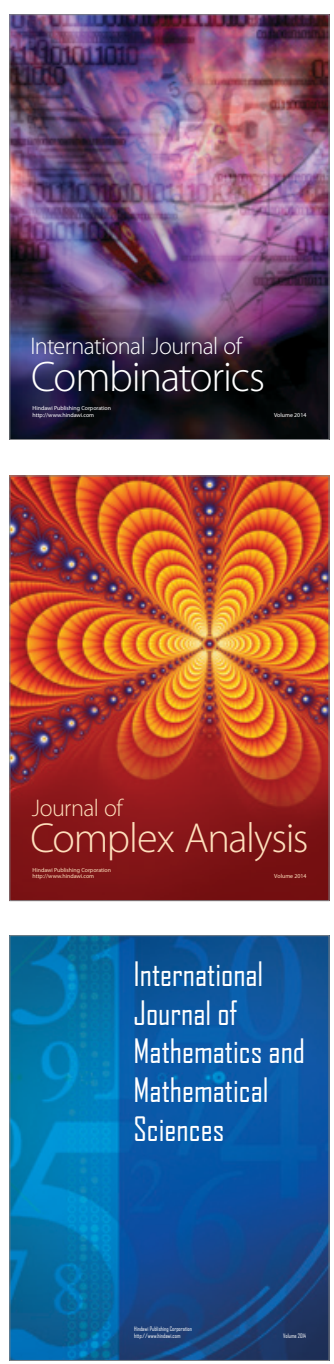
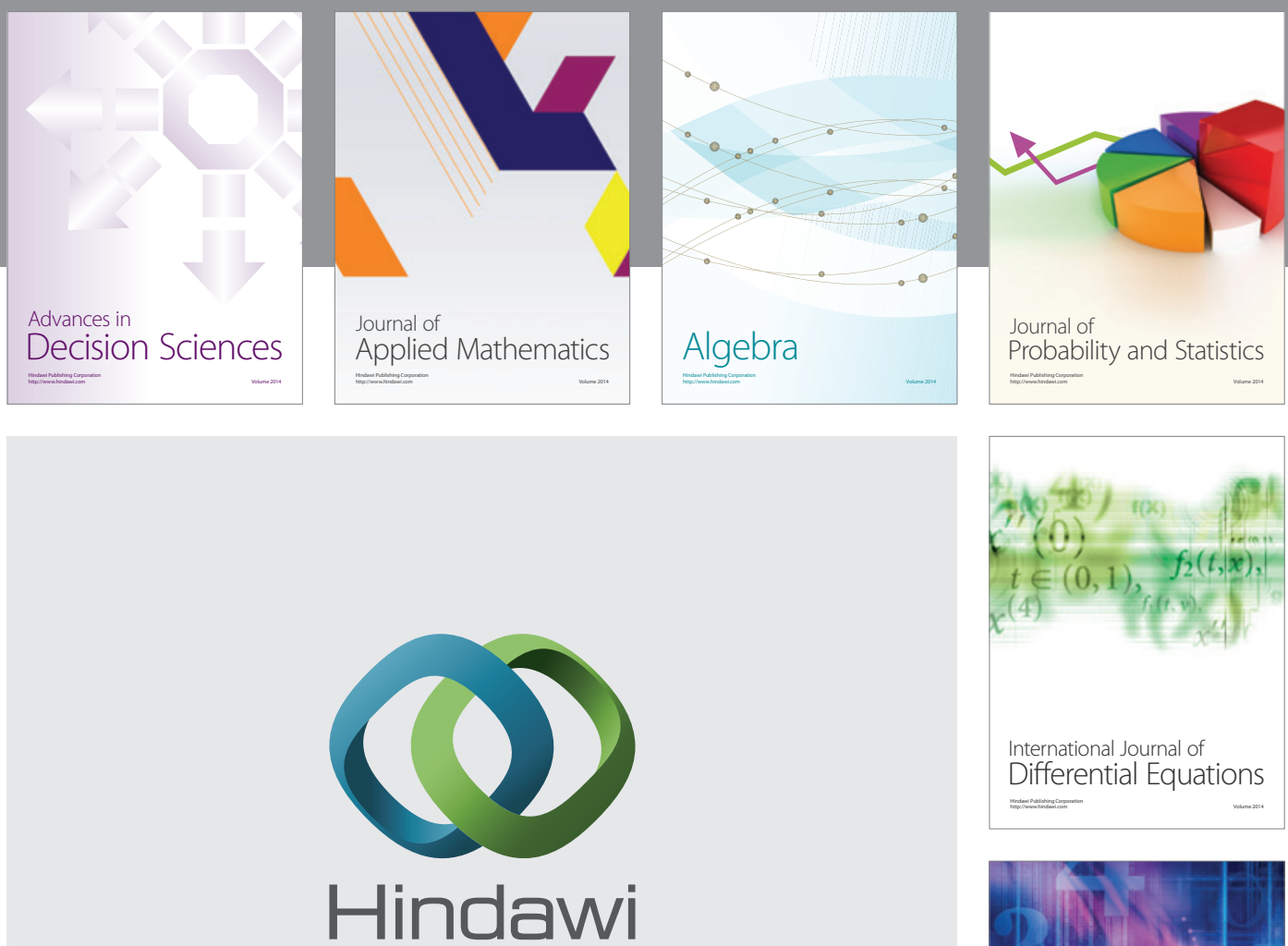

Submit your manuscripts at http://www.hindawi.com
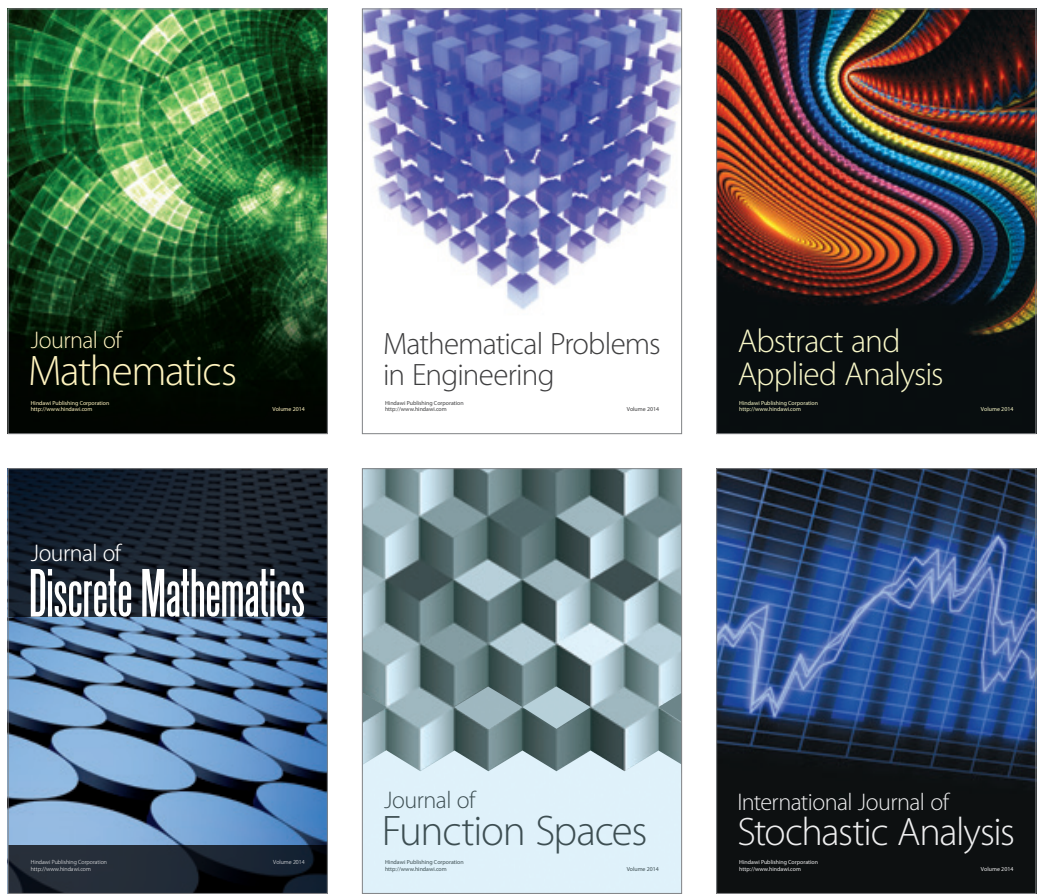

Journal of

Function Spaces

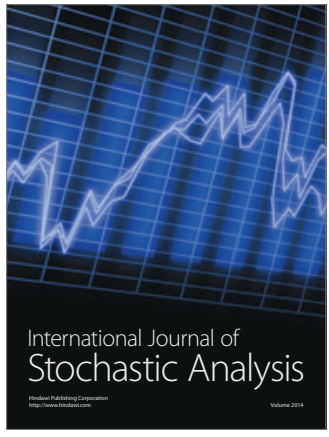

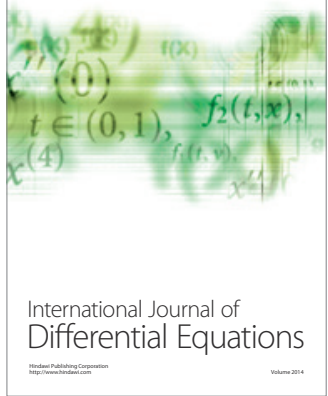
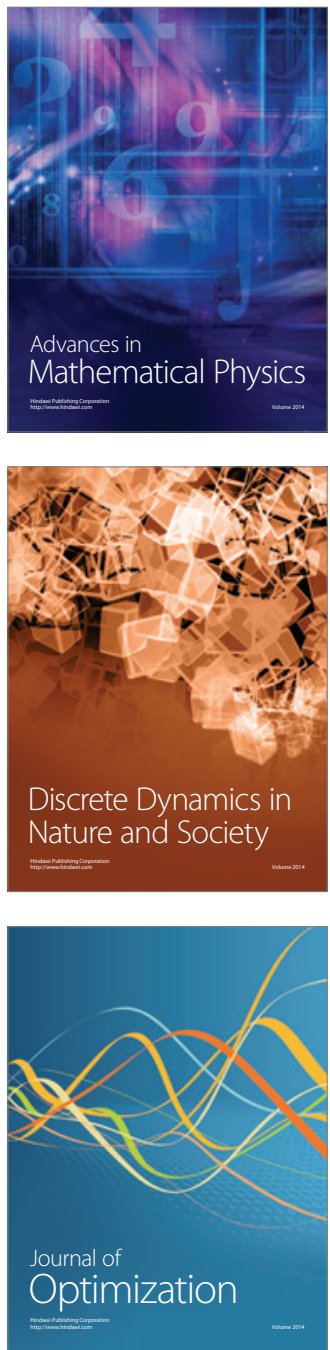